\title{
Optical sensitisation of energetic crystals with gold nanoparticles for laser ignition
}

\author{
Xiao Fang*, Mishminder Sharma, Christopher Stennett and Philip P Gill \\ Centre for Defence Chemistry, Cranfield University, Defence Academy of the UK, \\ Shrivenham SN6 8LA, UK.
}

*Corresponding author. Email: x.fang@cranfield.ac.uk

\begin{abstract}
The laser ignition of explosives is safer and more environmentally beneficial than electric ignition systems, but optical sensitisers must be present to facilitate heating by optical absorption. We investigated, for the first time, the optical sensitisation of cyclotrimethylenetrinitramine (RDX) crystals by doping them with gold nanoparticles to enhance laser ignitibility using a near-infrared diode laser. RDX crystals physically coated with gold nanoparticles or recrystallised from a solution containing gold nanoparticles were tested for optical sensitisation by micro-imaging with a scanning electron microscope, and high-speed video was used to experimentally observe the enhanced laser ignitibility. The gold nanoparticles achieved effective optical sensitisation and significantly enhanced the laser ignitibility of RDX, reducing the laser ignition threshold power from more than $45 \mathrm{~W}$ to as little as $1 \mathrm{~W}$. Our results show that gold nanoparticles are effective as optical sensitisers for the ignition of energetic materials using a small, low-power diode laser.
\end{abstract}

Keywords: gold nanoparticles; laser ignition; explosives; RDX crystallisation

\section{Introduction}

Explosive ignition by laser irradiation has been investigated for different types of primary and secondary explosives using different wavelengths and types of laser [1-8]. Laser ignition offers the prospect of safer, cleaner and more environmentally friendly ignition systems by replacing current electric ignitors that rely on toxic chemicals (e.g. lead azide) and/or sensitive primary explosives. Reliable ignition has been achieved with low-power lasers, enabling miniaturisation and cost reductions. The laser power required to ignite energetic materials was reduced by mixing energetic materials with optical sensitisers such as carbon black [1-2, 9-13]. The addition of $\sim 3 \%(\mathrm{w} / \mathrm{w})$ carbon black powder significantly increases the optical absorption and thus the laser ignitibility of the energetic materials. For example, mixing FOX-7 and hexanitrostilbene with carbon black for optical sensitisation greatly improved their near-infrared (NIR) absorption characteristics [1,2].

The laser irradiation of explosives causes heating by optical absorption, resulting in the formation of hotspots at discontinuities and inclusions present in the explosive material. This is considered the dominant mechanism responsible for laser ignition as indicated by the ignition of high-explosive nitrogen materials using xenon flash and $\mathrm{CO}_{2}$ lasers [3, $4,14,15]$. The increase in temperature of explosive materials accelerates exothermic decomposition, eventually achieving a sufficiently high temperature to ignite the explosive. Optical sensitisation has made explosives ignitable at various laser wavelengths, even within the NIR region where explosive materials have little optical absorption. Therefore, small and inexpensive infrared diode lasers could become the most suitable power sources for miniature laser ignition devices. 
In addition to common optical sensitisers such as carbon black, nanoparticles have been used as additives in energetic materials to enhance their optical and thermal properties and thus their ignition performance $[3,6,16,17]$. Carbon nanotubes are promising optical sensitisers because of their strong optical absorptivity and unique optical behaviour [3]. Pentaerythritol tetranitrate (PETN) sensitised with nickel nanoparticles can be ignited using a pulsed YAG laser, and it may be possible to modify the sensitivity of PETN by controlling the addition of such particles [6].

The phenomenon of surface plasmon resonance (SPR) allows metallic nanoparticles to be used as effective optical sensitisers, e.g. aluminium nanoparticles can enhance localised heating due to SPR [17]. Nanoparticles exposed to electromagnetic (EM) radiation with a wavelength longer than the particle diameter demonstrate an SPR phenomenon in which conduction-band electrons oscillate coherently, leading to strong EM waves on the particle surfaces and enhanced optical absorption and scattering. The strongly absorbed optical energy at a specific wavelength band is quickly converted to heat via a series of nonradiative processes over the particle surfaces $[17,18]$. The enhanced ability to absorb and quickly convert EM energy into heat makes plasmonic nanoparticles suitable as optical sensitisers. Gold is ideal as an additive in energetic materials because it is chemically stable and therefore more compatible and less toxic than other metals. During the laser ignition of explosives doped with gold nanoparticles (GNPs), the incident laser radiation induces a large local EM field around the GNPs in contact with the explosives [19-21] due to the coupling of laser radiation into an SPR, or a collective oscillation of the conductionband electrons $[22,23]$. When the SPR frequency band is comparable to that of the igniting laser, the GNPs strongly absorb at the laser wavelength and efficiently convert the absorbed energy into heat, producing dense nano-hotspots. Therefore, the ignitibility of explosives is expected to reach an optimum at the specific laser wavelength. The optimisation of SPR absorbance mainly depends on the size and density of the nanoparticles [24,25], and can be tuned to a desirable laser wavelength to enhance laser ignitibility and wavelength selectivity.

Here we experimentally investigated the laser ignition of micro-energetic cyclotrimethylenetrinitramine (RDX) crystals doped with GNPs, aiming to greatly enhance their laser ignitibility. An 808-nm diode laser was used as the igniting power source, and the GNPs demonstrated maximum SPR and thus an optimal optical absorption band at this wavelength. RDX was chosen because it is widely used in explosive products such as propellants, boosters, detonators and the main charge filling compositions of warheads.

\section{Method and experimental}

\subsection{Sample materials}

RDX type 1 was used as the high explosive for optical sensitisation. Analytical grade acetone (Sigma-Aldrich Ltd., UK) was used as the solvent to recrystallise the RDX. Rodshaped GNPs, $10 \mathrm{~nm}$ in diameter and $41 \mathrm{~nm}$ in length (Nanopartz, USA), achieve strong optical absorption at $808 \mathrm{~nm}$ and were chosen as the optical sensitisers (Figure 1). The citrate-capped GNPs were dispersed in deionised water at a concentration of $3.5 \mathrm{mg} / \mathrm{ml}$. The capping agent was present to prevent nanoparticle aggregation.

\subsection{Sample preparation and characterisation}

Three types of RDX crystal samples were prepared using different processing methods: 
S1 - Pure RDX (Type I) crystals recrystallised from RDX-acetone solution mixed with deionised water;

S2 - $0.5 \%$ (w/w) GNP-coated RDX (Type I) obtained by physically mixing pure RDX crystals (S1) with GNPs;

S3 - 0.5\% (w/w) GNP-doped RDX (Type I) recrystallised from RDX-acetone solution mixed with GNP dispersions in deionised water.

\subsubsection{Re-crystallisation}

RDX re-crystallisation was achieved by a crystallization mechanism based on cooling and evaporation. To obtain S1, $400 \mathrm{mg}$ of RDX powder was weighed in a vial and $4 \mathrm{ml}$ acetone was added. The vial was sealed with a seal cap and heated in an oil bath at $\sim 60^{\circ} \mathrm{C}$ until the RDX powder was completely dissolved. Deionised water $(1 \mathrm{ml})$ at room temperature was mixed with the RDX-acetone solution in the vial, which induced a mild crash (or precipitation) into the solution. The vial was sealed with film containing several pinholes and left to cool and evaporate naturally. The sample was nearly dry after 4 days and was placed in a vacuum oven at $100^{\circ} \mathrm{C}$ for $1 \mathrm{~h}$ to dry completely. Pure RDX crystals (S1) were obtained ready for use. The size of the crystals ranged from sub-millimetre to 1-2 mm (Figure 2a). RDX doped with GNPs (S3) was prepared in a similar manner to S1, but the deionised water was replaced with $1 \mathrm{ml}$ of the GNP dispersion and $3 \mathrm{ml}$ RDX-acetone solution containing $300 \mathrm{mg} \mathrm{RDX}$ was added after mixing (Figure 2c).

\subsubsection{Physical mixing}

Pure RDX crystals ( 350 $\mathrm{mg}$ of $\mathrm{S} 1$ ) were weighed into a glass vial and $0.5 \mathrm{ml}$ of the GNP dispersion was dispensed into the vial. The slurry was mixed to ensure the explosive crystals and the gold particles were dispersed homogeneously. The vial was left in a fume cupboard to allow the water to evaporate. The explosive mix was stirred daily to prevent caking and settlement of the GNPs before they were dry. When the sample was nearly dry it was placed in a vacuum oven at $100^{\circ} \mathrm{C}$ to dry completely. This crystal sample (S2) of RDX coated with GNPs had the same doping concentration of $0.5 \%(\mathrm{w} / \mathrm{w})$ as $\mathrm{S} 3$ and was then ready to use, as shown in Figure $2 b$.

\subsubsection{Sample characterisation}

Differential scanning calorimetry (DSC) was carried out using a DSC-30 device (Mettler Toledo) to investigate any potential differences in the thermal properties of the RDX crystal samples. The heating temperature range was $30-300^{\circ} \mathrm{C}$ increasing at a rate of $10^{\circ} \mathrm{C} / \mathrm{min}$. We used 2-mg samples of S1 and S3 for analysis. The prepared RDX crystal samples were also visualised by scanning electron microscopy (SEM) using a FEI XL30-SFEG instrument (Thermo Fisher Scientific) capable of resolutions higher than $2 \mathrm{~nm}(600,000 \mathrm{x}$ magnification) and including an energy dispersive X-ray spectroscopy (EDS) system for elemental analysis. The crystal samples were mounted on a sample stab $(\sim 12 \mathrm{~mm}$ diameter) and the locations of the individual crystals were labelled in order to allocate each of them during SEM analysis.

\subsection{Experimental setup}

The experimental setup for the laser ablation/ignition of an unconfined crystal sample is shown in Figure 3. The diode laser (JENOPTIK JOLD-X-CPXF) provided power of up to 45 $\mathrm{W}$ at $808 \mathrm{~nm}$ and was operated in continuous-wave (CW) mode. Each crystal was placed 
on an aluminum plate sample holder and the laser beam was focused onto the surface using a lens $50 \mathrm{~mm}$ in diameter with a $50-\mathrm{mm}$ focal length. The diameter of the laser spot on the sample was $\sim 1.5 \mathrm{~mm}$, comparable to the sample size. The light signals from the laser and the ignited flame were detected using a photodiode detector (OSRAM Silicon PIN Photodiode BPX 65) and recorded with a $600 \mathrm{MHz}$ digitising oscilloscope (Hewlett Packard 5411D). The time difference between the origins of the two signals was measured as the ignition delay time. A high speed camera (Casio EX850) capable of recording up to 1000 frames per second was fitted on an optical rail to observe the side of the sample. Each crystal was irradiated by the laser beam on its upper surface.

\section{Results and discussion}

\subsection{DSC analysis}

DSC was carried out to investigate the thermal properties of the samples and to ensure the energetic material was not chemically modified by doping/mixing with the inert GNPs (Figure 4). The RDX samples analysed gave a single endothermic onset peak at around $205^{\circ} \mathrm{C}$. This was from the melting of the RDX prior to its thermal decomposition. Subsequently there was a single larger exotherm due to the decomposition of RDX with a peak at approximately $240^{\circ} \mathrm{C}$. This exothermic peak tended to be wide. There was no notable change when comparing samples S1 and S3 in terms of the onset temperatures $\left(204.9^{\circ} \mathrm{C}\right.$ and $\left.205.5^{\circ} \mathrm{C}\right)$ or the peak temperatures $\left(207.3^{\circ} \mathrm{C}\right.$ and $208.3^{\circ} \mathrm{C}$ for melting, $239.7^{\circ} \mathrm{C}$ and $240.7^{\circ} \mathrm{C}$ for decomposition, respectively). This data indicates the RDX mixed with the GNPs was chemically compatible.

\subsection{SEM analysis}

SEM images were prepared for samples S2 and S3 to visualise the effects of GNP doping. The SEM image of sample S3 and the energy dispersive spectrum (EDS) of the area enclosed by the white square are shown in Figure 5. The GNPs were present on the RDX crystal surface as revealed by the bright patches in the SEM image and the EDS spectrum revealed the gold composition of the white area. SEM imaging of surfaces created by crushing the S3 crystals was carried out to observe the internal matrix (Figure 6). Clusters of GNPs, shown as bright inclusions, were also present inside the crystal. Therefore, recrystallisation from an RDX-acetone solution mixed with GNPs dispersed in water allows the doping of RDX crystals with GNPs both on the surface and within the crystal. When heated by laser, the GNP inclusions should form hotspots inside the crystal, and would be expected to transfer heat to the surrounding RDX more efficiently than GNPs on the surface due to the larger interfacing area between the GNPs and RDX and the absence of direct heat dissipation into the air. Laser heating and the resulting exothermal decomposition inside the RDX crystal would also rapidly induce local pressure surrounding the GNPs, and thus increase the temperature and impact of these hotspots.

For comparison, the SEM image of sample $\mathrm{S} 2$ prepared by physical mixing is shown in Figure 7a. The image shows a more uniform distribution of the GNPs (white spots) on the sample surface compared to the distribution in Figure $5 \mathrm{a}$. This suggests there was little or no aggregation of the GNPs when they were mixed with RDX crystals in deionised water during sample preparation, whereas the GNPs tended to aggregate when they were provided as an RDX-acetone solution in the recrystallisation method. This useful information could facilitate the uniform doping of RDX with GNPs by optimising the solvent ratio in the GNP dispersion. During SEM imaging, the RDX crystal surface was easily damaged by a focused electron beam when high-resolution imaging for nano-scale image 
analysis was attempted. Such damage is revealed by the cracks in the SEM image shown in Figure 7b. Therefore, nano-scale details of the RDX crystals could not be recorded, although the SEM system we used was capable of such high-resolution imaging.

\subsection{Laser ignitibility}

\subsubsection{Laser ignition threshold}

To evaluate the laser ignitibility of GNP-sensitised RDX energetic crystals, laser ignition tests were carried out at various laser powers for samples S1, S2 and S3. Ignition success rates (\%) were defined as the number of successful ignition events with a single shot among seven repeated ignition attempts at the same laser power. The results at various laser powers for the three samples are shown in Table 1. Defined as greater than $50 \%$ ignition success rate, the laser ignition thresholds were $\sim 1 \mathrm{~W}$ for S3 and $\sim 1.8 \mathrm{~W}$ for S2. No ignition was achieved for sample S1 (pure RDX crystals) at any laser power up to the maximum available power of $45 \mathrm{~W}$.

The RDX crystals doped with GNPs were more optically sensitive than crystals with GNPs coated on the surface. This reflects the more efficient heating of RDX crystals achieved by the embedded GNPs, which can disperse heat from their entire surface area rather than the limited area in contact with the RDX in the case of surface GNPs. The confined hotspots achieve a high local gas pressure to interact with the surrounding RDX, achieving a greater than 45 -fold increase in laser ignitibility or ignition sensitivity.

Table 1 Laser ignition thresholds for RDX crystal samples.

\begin{tabular}{|l|l|l|l|l|l|}
\hline \multirow{2}{*}{ Sample type } & \multicolumn{5}{|l|}{ Percentage ignition at laser powers } \\
\cline { 2 - 6 } & $\mathbf{3}$ W & $\mathbf{2}$ W & $\mathbf{1 . 8}$ W & $\mathbf{1 . 5}$ W & $\mathbf{1 ~ W}$ \\
\hline $\begin{array}{l}\text { S1 } \\
\text { Pure RDX crystal }\end{array}$ & \multicolumn{5}{|l|}{ No ignition at laser powers of up to 45 W } \\
\hline $\begin{array}{l}\text { S2 } \\
\text { 0.5\% (w/w) GNP- } \\
\text { coated crystal }\end{array}$ & $\begin{array}{l}100 \% \\
(7 / 7)\end{array}$ & $\begin{array}{l}100 \% \\
(7 / 7)\end{array}$ & $\begin{array}{l}57 \% \\
(4 / 7)\end{array}$ & $\begin{array}{l}29 \% \\
(2 / 7)\end{array}$ & $\begin{array}{l}0 \% \\
(0 / 7)\end{array}$ \\
\hline $\begin{array}{l}\text { S3 } \\
\begin{array}{l}\text { O.5\% (w/w) GNP- } \\
\text { doped crystal }\end{array}\end{array}$ & $\begin{array}{l}100 \% \\
(7 / 7)\end{array}$ & $\begin{array}{l}100 \% \\
(7 / 7)\end{array}$ & $\begin{array}{l}100 \% \\
(7 / 7)\end{array}$ & $\begin{array}{l}86 \% \\
(6 / 7)\end{array}$ & $\begin{array}{l}57 \% \\
(4 / 7)\end{array}$ \\
\hline
\end{tabular}

\subsubsection{Laser ignition map}

Laser ignition tests were carried out on samples S2 and S3 to study the effect of applied laser power on the ignition delay, which is the time from initial laser exposure to the onset of the ignited burn. The delay times at various ignition laser powers were measured and are plotted with trend lines in Figure 8. The plot, also known as an 'ignition map', indicates how quickly the ignition took place at various laser powers. The plots for both of the samples show that as the ignition power increases the ignition delay declines sharply at lower-medium powers (e.g. $<20 \mathrm{~W}$ ) and tends towards saturation level at higher powers. At the maximum power ( $45 \mathrm{~W}$ ), the ignition delay was reduced to $\sim 2.1 \mathrm{~ms}$ for $\mathrm{S} 2$ and 1.3 ms for S3. Therefore, the GNP-doped RDX crystal is more sensitive than the GNP-coated crystal in terms of the ignition delay, as shown previously for the ignition threshold. 


\subsection{Effect of laser irradiation on GNP-sensitised RDX crystals}

\subsubsection{Laser power below the ignition threshold}

To study the effect of laser irradiation on the GNP-doped RDX crystals, sample S3 was exposed to laser radiation at a power below the ignition threshold. Figure 9 shows the SEM images of the crystals after irradiation at $0.4 \mathrm{~W}$ (Figure 9b) and $0.8 \mathrm{~W}$ (Figure 9d). It indicates that the crystals were broken up by laser ablation at both powers, with the higher power initiating more cracking and splitting. The laser also caused surface stripping and smoothening, again with more damage at the higher power. These results can be explained by the GNPs within the crystal absorbing the applied laser energy efficiently and instantly becoming hotspots confined within the crystal. The resulting internal heat and pressure cause the cracking and splitting. The damage patterns are probably dependent on the distribution of GNPs in the crystal, arising naturally during recrystallisation. The subsurface and surface GNPs in the same crystal would also absorb laser radiation, being responsible for the surface stripping and smoothening. The optical sensitisation of the S3 sample was thus initiated both inside the body of the crystal and on the surface.

Similar tests on the S2 crystals did not cause cracking or splitting, but did cause surface ablation effects (Figure 10a-d). These results are consistent with the tests on sample S3 because the absence of internal GNPs would prevent heat and pressure inside the crystal causing fractures, but the surface damage in samples S2 and S3 is similar because both contain GNPs on or near the surface where optical absorption occurred at the NIR laser wavelength. In Figure $10 \mathrm{~b}$, the crystal surface was irradiated at $0.4 \mathrm{~W}$ and appears pitted due to the laser-induced heating of the GNPs on the surface. In Figure 10d, the crystal surface was irradiated at $0.8 \mathrm{~W}$ and there is evidence of surface melting. The S2 crystal was therefore optically sensitised by the GNPs on its surface.

\subsubsection{Laser power at the ignition threshold}

To analyse the ignition behaviour of RDX crystals doped or coated with GNPs, samples S2 and S3 were ignited by laser radiation at the sufficient powers of 1 and $2 \mathrm{~W}$, respectively. Images of the ignition process were captured at 1000 frames per second. Figure 11a,b shows the image series for two individual S3 crystals before and during laser ignition. The laser was directed vertically onto the crystal, with the impact point at the top of each image. After $13 \mathrm{~ms}$ (Figure 11a), the crystal has ignited and both the top and bottom surfaces begin to burn. After $21 \mathrm{~ms}$, micro-explosions have occurred at the bottom, with the induced shock waves pushing the crystal up from its original position. At $38 \mathrm{~ms}$, violent micro-explosions inside the crystal create micro-fragments that shatter outwards. Finally, the post-explosion reside is ejected from the laser spot after $50 \mathrm{~ms}$. Figure $11 \mathrm{~b}$ shows the sequence of events for another S3 crystal. Ignition starts at $\sim 21 \mathrm{~ms}$, internal microexplosions begin at $46 \mathrm{~ms}$ causing outward shattering, and again the post-explosion reside is ejected from the laser spot after $50 \mathrm{~ms}$. In contrast to the S3 crystals, the ignition of S2 crystals coated with GNPs initiates on the surface, and this surface begins to burn after 14 ms (Figure 11c). The burn develops further along the laser beam through the crystal (35$63 \mathrm{~ms}$ ), and a full burn (deflagration) of the crystal occurs at $\sim 70 \mathrm{~ms}$. This fast burning process occurred without micro-explosions, as anticipated due to the presence of GNPs solely on the crystal surface.

These data confirm that the ignition of S3 crystals involves internal micro-explosions that cause rapid splitting, which can be attributed to the presence of GNPs inside the crystal and its sub-surface. These confined GNPs absorb laser energy to become hotspots, which 
increase the surrounding local temperature of the RDX crystal beyond its ignition level and initiate exothermal reactions (e.g. decomposition and vaporisation) that are confined in the crystal. These reactions generate pressure against the inner walls of the crystal around the hotspots which is released as a shock wave that propagates through the crystal. The analysis of several S3 crystals (two examples are shown in Figure 11) indicates that the burn and micro-explosions are initiated at different locations in the crystal and with different delay times and strengths. Such variability may be dependent on the distribution of GNPs or the associated hotspots within the RDX crystal. The mechanism of photosensitisation by GNP doping inside an energetic crystal may therefore not only greatly enhance its laser ignitibility but also induce useful shock wave and impact forces that initiate the main charge explosives.

\section{Conclusions}

This study has, for the first time, experimentally confirmed the feasibility and effectiveness of GNPs as optical sensitisers for RDX crystals in the context of laser ignition at $808 \mathrm{~nm}$. The threshold laser power requirement for the ignition of RDX crystals was lowered by at least 45-fold. GNP-doped RDX crystals (S3) produced by RDX re-crystallisation confirmed that laser-induced ignition was initiated from both the surface and inner matrix of the crystal, with deflagration and micro-explosions taking place during the ignition process. Such RDX crystals were superior in terms of optical sensitisation to GNP-coated RDX crystals (S2) produced by simple mixing. The ignition of the latter was initiated from the surface alone. Therefore, RDX re-crystallisation from GNP dispersions is a better sensitisation method for the laser ignition of RDX. The threshold laser power was reduced to $\sim 1 \mathrm{~W}$, which achieved the reliable ignition of GNP-sensitised RDX crystals in more than $50 \%$ of attempts, in contrast to pure RDX crystals without sensitisers which could not be ignited even with the highest available laser power of $45 \mathrm{~W}$. Optically-sensitised energetic crystals produced using this innovative method allow the efficient utilisation of laser-heated GNPs by heating the crystal matrix, and further investigation should therefore facilitate the development of miniature low-power laser ignition systems.

\section{Acknowledgements}

The authors would like to thank Dr Ranko Vrcelj for his valuable advice on sample preparation and Xian Wei and Christine Kimpton for support with the SEM analysis.

\section{References}

[1] X. Fang, S.R. Ahmad, Laser ignition of an optically sensitized secondary explosive by a diode laser, Central European Journal of Energetic Material 13 (2016) 103115.

[2] X. Fang, W.G. Mcluckie, Laser ignitibility of insensitive secondary explosives 1,1diamino-2,2-dinitroethene (FOX-7), Journal of Hazardous Materials 285 (2015) 375-382.

[3] N.N. De, N.R. Cummock, I.E. Gunduz, B.C. Tappan, Photoflash and laser ignition of select high-nitrogen materials, Combustion and Flame 167 (2016) 207-217.

[4] J.A. Brown, D.A. LaBarbera, M.A. Zikry, Laser interaction effects of electromagnetic absorption and micro structural defects on hot-spot formation in RDX-PCTFE energetic aggregates, Modell. Simul. Mater. Sci. Eng. 22 (2014) 55013 (22pp). 
[5] Z. Yan, C. Zhang, W. Liu, J. Li, M. Huang, X. Wang, G. Zhou, B. Tan, Z. Yang, Z. $\mathrm{Li}$, L. Li, H. Yan, X. Yuan, X. Zu, Ultraviolet laser-induced ignition of RDX single crystal, Scientific Reports 6 (2016) 20251, DOI: 10.1038/srep20251.

[6] B.P. Aduev, D.R. Nurmukhametov, R.I. Furega, I.Y. Liskov, Initiation of explosion of Pentaerythritol Tetranitrate by pulses of the first and second harmonics of a Neodymium laser, Russian Physics Journal 58 (2015) 1093-1097.

[7] A.M. Renlund, P.L. Stanton, W.M. Trott, Laser initiation of secondary explosives, Proc. $9^{\text {th }}$ Int. Detonation Symp. (1989) 1118-1127.

[8] J.T. Hagan, M.M. Chaudhri, Low energy initiation of single crystals $\beta$-lead azide, Journal of material science 16 (1981) 2457-2566.

[9] D. Damm, M. Maiorov, Thermal and radiative transport analysis of laser ignition of energetic materials, Proc. of SPIE 7795 (2010) 779502.

[10] D. Ewick, Laser initiated detonator - recent developments, 33rd AIAA/ASME/SAE/ASEE Joint Propulsion Conference \& Exhibit (1997).

[11] A.C. Munger, T.M. Beckman, D.P. Kramer, Hot-wire versus laser, is there a difference?, 18th Int. Pyrotechnics Seminar, Colorado (1992).

[12] R.D. Skocypec, A.R. Mahoney, M.W. Glass, R.G. Jungst, N.A. Evans, K.L. Erikson, Modelling laser ignition of explosives and pyrotechnics: effects and characterisation of radiative transfer, 15th Int. Pyrotechnics Seminar, Boulder (1990).

[13] J.M. Zucker, B.C. Tappan, D.M. Oschwald, D.N. Preston, N.J. Burnside, Characterisation of energetic formulations optimised for optical initiation, Proc. $14^{\text {th }}$ Int. Detonation Symp. (2010) 724-729.

[14] M.W. Chen, S.Y. Kenneth, D. Suslick, Hot spot generation in energetic materials created by long-wavelength infrared radiation, Appl. Phys. Lett. 104 (2014) 061907.

[15] M.W. Chen, S.Y. Kenneth, S. Suslick, D.D. Dlott, Hot spots in energetic materials generated by infrared and ultrasound, detected by thermal imaging microscopy, Rev. Sci. Instrum. 85 (2014) 023705.

[16] J.J. Granier, T. Mullen, M.L. Pantoya, Non uniform laser ignition in energetic materials, Combustion Science and technology 175 (2003) 1929-1951.

[17] J. E. Abboud, X. Chong, M. Zhang, Z. Zhang, N. Jiang, S. Roy, J.R. Gord, Photothermally activated motion and ignition using aluminium nanoparticles, Applied Physics Letters 102 (2013) 023905.

[18] M. A. El-Sayed, X. Huang, Gold nanoparticles: Optical properties and implementations in cancer diagnosis and photothermal therapy, Journal of Advanced Research 1 (2010) 13-28.

[19] Y. Cui, Y. He, Y. Jin, F. Ding, L. Yang, Y. Ye, Plasmonic and metamaterial structures as electromagnetic absorbers, Laser and Photonics Reviews 8 (2014) 495-520.

[20] M. Schnell, P. Alonso-González, L. Arzubiaga, F. Casanova, L.E. Hueso, A. Chuvilin, R. Hillenbrand, Nanofocusing of mid-infrared energy with tapered transmission lines, Nat. Photonics 5 (2011) 283-287.

[21] H. Cang, A. Labno, C. Lu, X. Yin, M. Liu, C. Gladden, Y. Liu, X. Zhang, Probing the electromagnetic field of a 15-nanometre hotspot by single molecule imaging, Nature 469 (2011) 385-388.

[22] M.M. Alvarez, J.T. Khoury, T.G. Schaaff, M.N. Shafigullin, I. Vezmar, R. L. Whetten, Optical absorption spectra of nanocrystal gold molecules, J. Phys. Chem. B 101 (1997) 3706-3712. 
[23] L. S. Jung, C. T. Campbell, T.M. Chinowsky, M.N. Mar, S.S. Yee, Quantitative interpretation of the response of surface plasmon resonance sensors to adsorbed films, Langmuir 14 (1998) 5636-5648.

[24] K. Kurihara, K. Suzuki, Theoretical understanding of an absorption-based surface plasmon resonance sensor based on Kretchmann's theory, Anal. Chem. 74 (2002) 696-701.

[25] T.A. El-Brolossy, T. Abdallah, M.B. Mohamed, S. Abdallah, K. Easawi, S. Negm, $\mathrm{H}$. Talaat, Shape and size dependence of the surface plasmon resonance of gold nanoparticles studied by Photoacoustic technique, Eur. Phys. J. Special Topics 153 (2008) 361-364. 

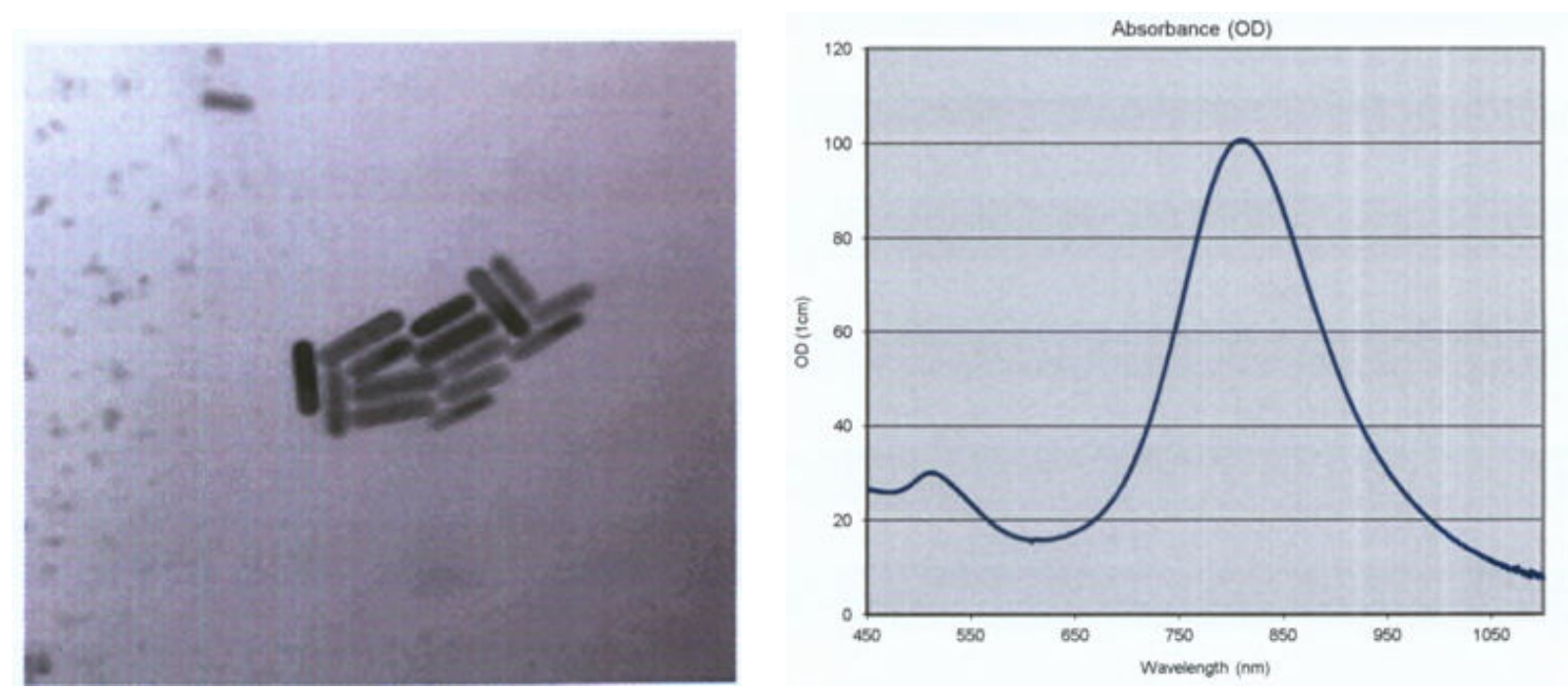

Figure 1 Scanning electron micrograph and absorption spectrum of the GNPs used in this study, which were provided by the manufacturer (Nanopartz, USA).
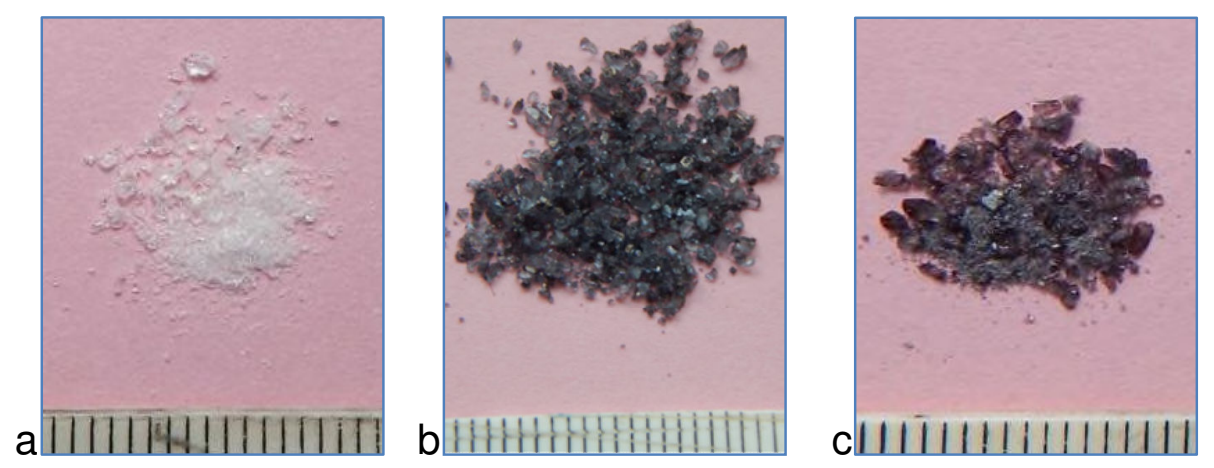

Figure 2 RDX preparations (gradations are in millimetres): a) S1, pure RDX crystals.

b) S2, mixture of RDX crystals and GNPs. c) S3, recrystallised RDX doped with GNPs.

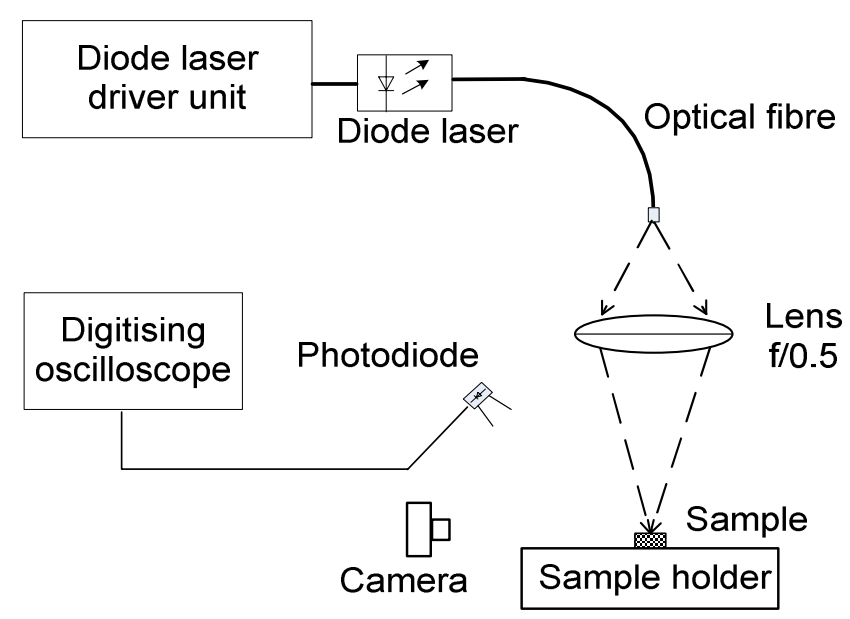

Figure 3 Experimental setup for laser ablation/ignition of a small energetic crystal. 

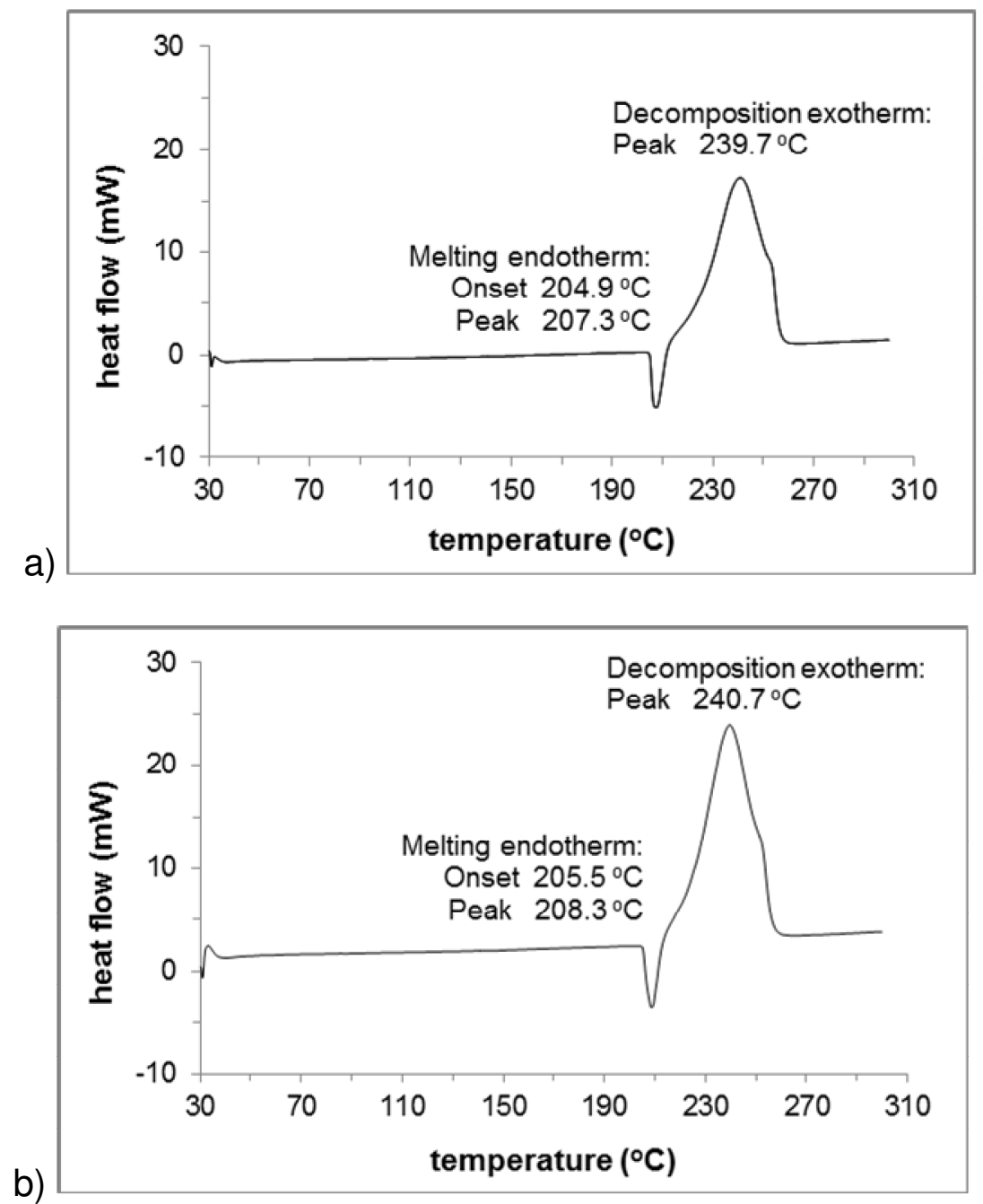

Figure 4 DSC curves of a) pure RDX crystals (S1), and b) RDX crystals doped with GNPs (S3).

a)

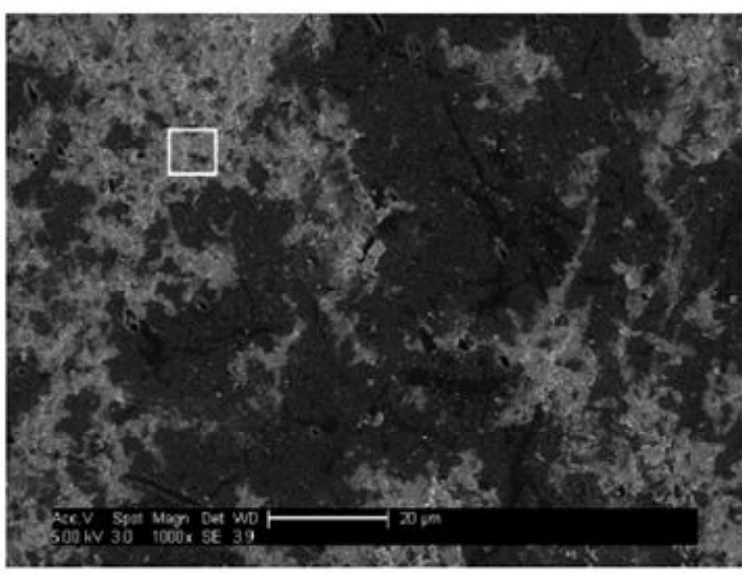

b)

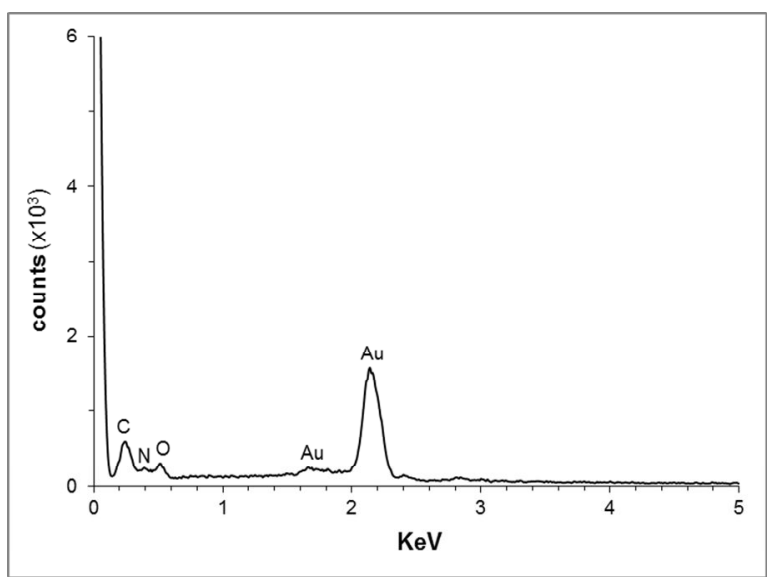

Figure 5 The crystal surface of RDX doped with GNPs (S3): a) SEM image, and b) EDS of the bright areas identified as GNPs. 


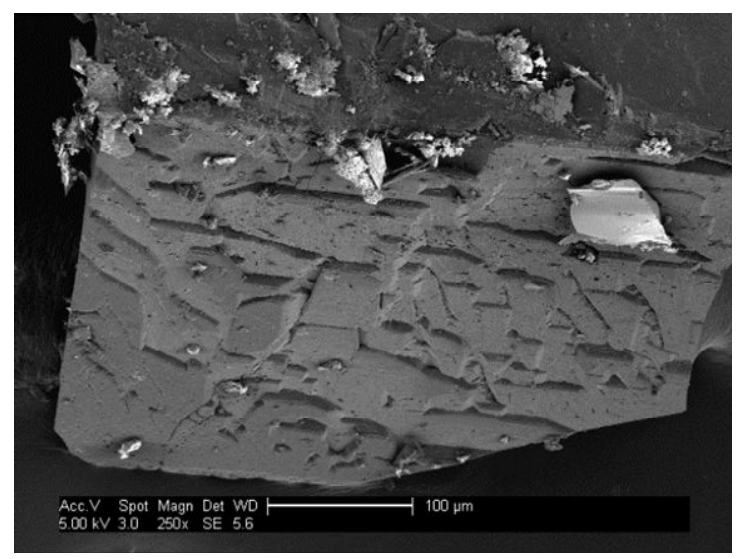

Figure 6 SEM image of the inner surface of a broken RDX crystal doped with GNPs.

a)

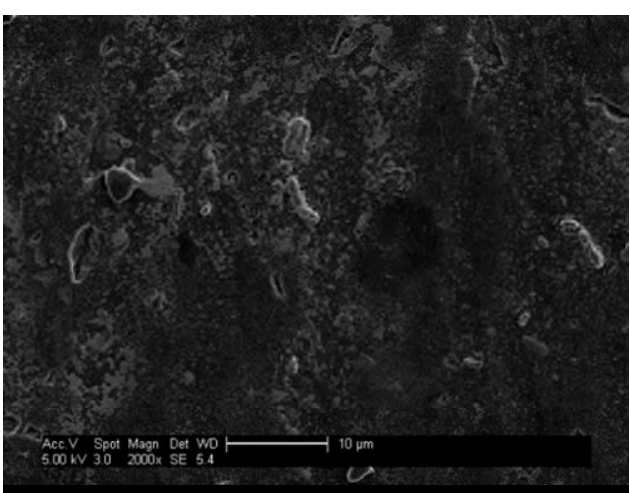

b)

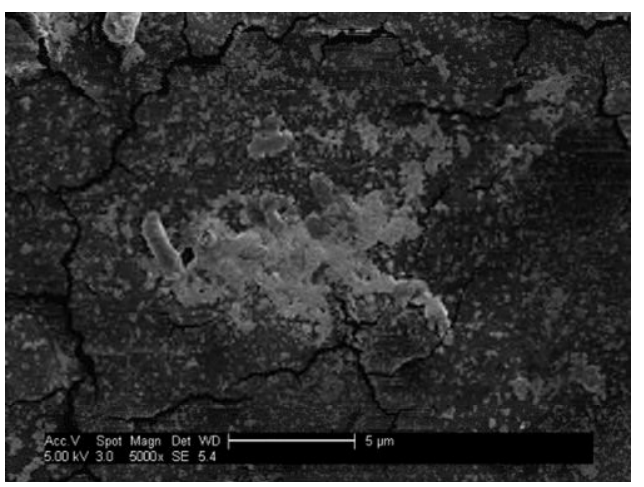

Figure 7 The crystal surface of RDX physically mixed with GNPs (S2): a) SEM image, and b) SEM image showing surface damage (cracks) caused by a tightly focused electron beam.

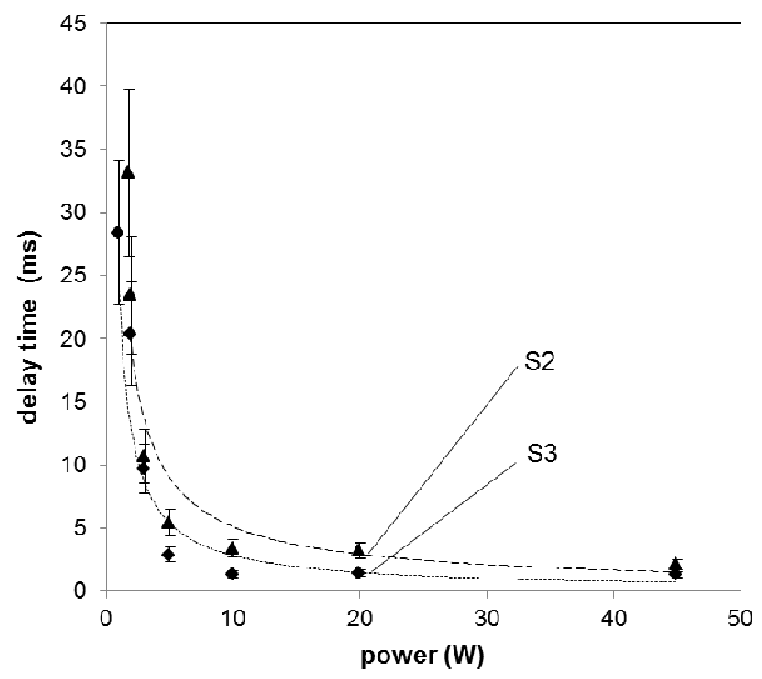

Figure 8 Ignition delay time vs. laser power for samples S2 and S3. Each measurement was averaged over seven tests at each laser power. The relative standard deviation was $\sim 20 \%$. 

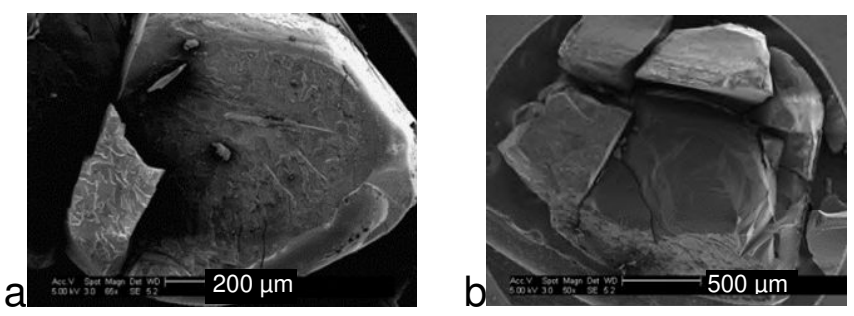

Figure 9 SEM images of S3 crystals after laser ablation at $0.4 \mathrm{~W}$ (a) and $0.8 \mathrm{~W}$ (b).
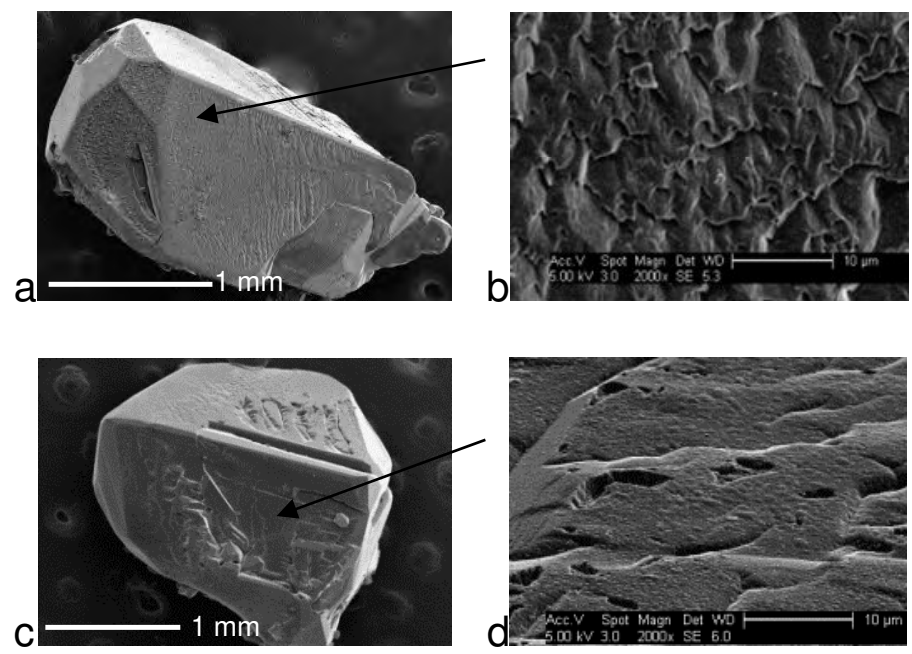

Figure 10 SEM images of laser-irradiated S2 crystals and their ablated surfaces at laser powers of $0.4 \mathrm{~W}(\mathrm{a}, \mathrm{b})$ and $0.8 \mathrm{~W}(\mathrm{c}, \mathrm{d})$.
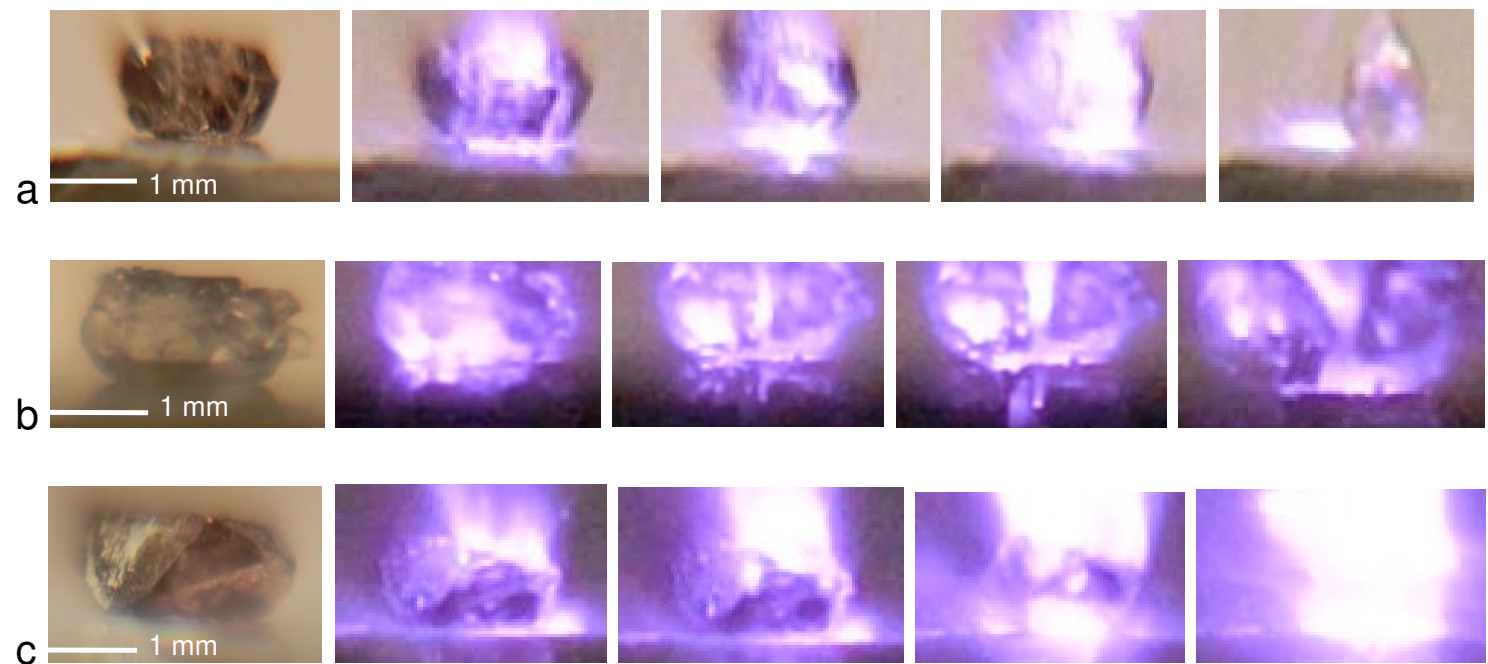

Figure 11 Time course of crystal ignition and burning: (a) S3 crystal at laser exposure times (left to right) $t=0,13,21,38,50 \mathrm{~ms}$. (b) $S 3$ crystal at exposure times (left to right) $t=0,21,46,48,50 \mathrm{~ms}$. (c) $S 2$ crystal at exposure times (left to right) $t=0,14,35,63,70$ ms. 